\title{
Genetic alteration of ARMC5 in a patient diagnosed with meningioma and primary macronodular adrenal hyperplasia: a case report
}

\author{
Teruo Jojima@1, Takahiko Kogai ${ }^{2}$, Toshie lijima', Kanako Kato', Masaaki Sagara', \\ Atsumi Kezuka', Masato Kase', Shintaro Sakurai', Kazumi Akimoto ${ }^{3}$, Junko Sakumoto², \\ Takashi Namatame ${ }^{3}$, Keisuke Ueki ${ }^{4}$, Akira Hishinuma ${ }^{2}$, Takao Kamai ${ }^{5}$, Isao Usui ${ }^{1}$ and \\ Yoshimasa Aso' \\ ${ }^{1}$ Department of Endocrinology and Metabolism, Dokkyo Medical University, ${ }^{2}$ Department of Infection Control and \\ Clinical Laboratory Medicine, ${ }^{3}$ Division of Clinical Science, Research Support Center, ${ }^{4}$ Department of Neurosurgery, \\ and ${ }^{5}$ Department of Urology, Dokkyo Medical University, Shimotsuga-gun, Tochigi, Japan
}

Correspondence should be addressed to T Kogai

Email

tkogai@dokkyomed.ac.jp

\begin{abstract}
A monoallelic germline alteration of $A R M C 5$ causes primary bilateral macronodular adrenal hyperplasia (PBMAH) with Cushing's syndrome via its subsequent somatic alteration on the other allele as the second hit. PBMAH is sometimes complicated with meningioma. Dependency of such a multi-organ disease on the second hit mechanism was reported before, but this finding has not been confirmed yet. We describe a case of a 65-year-old female with PBMAH, carrying a heterozygous germline alteration of ARMC5, p.R267*, complicated with meningioma associated with somatic loss of heterozygosity $(\mathrm{LOH})$ of the unaffected allele. Pathogenic alterations of $A R M C 5$ may also contribute to the development of meningioma by the two-hit mechanism.
\end{abstract}

\section{Introduction}

Primary bilateral macronodular adrenal hyperplasia (PBMAH) is a rare cause of subclinical or overt Cushing's syndrome (CS). Germline alterations of ARMC5 are frequently observed in $\mathrm{PBMAH}$, leading to downregulation of apoptosis in adrenocortical cells and subsequent increase of cortisol secretion (1). The abnormality of ARMC5 causes PBMAH via the 'two-hit mechanism, similarly to the tumor suppressor genes: a germline lossof-function alteration of ARMC5 as the first hit, followed by a second hit of a somatic alteration of ARMC 5 on the other allele, including loss of heterogeneity ( $\mathrm{LOH})$ or another heterozygous pathogenic variant (2).
Several tumor suppressor genes, such as BRCA, RET, and MEN1, cause multi-organ tumors, especially in the tissues abundantly expressing such a gene, dependent on the two-hit mechanism. Three pedigrees of PBMAH carrying abnormal ARMC5 have been reported with concomitant intracranial meningioma $(3,4,5)$. Since $A R M C 5$ is abundantly expressed not just in adrenal glands, but also in brain (6), the two-hit theory with ARMC5 alterations could be expanded to the development of concomitant brain tumor. Only one case of meningioma, however, has been so far reported for such a somatic alteration on the other allele (5). 
Here, we report a case of PBMAH with a concomitant meningioma, carrying a heterozygous germline alteration of p.R267* in ARMC5, a well-characterized pathogenic variant for PBMAH $(2,7,8)$, but also somatic $\mathrm{LOH}$ of the $A R M C 5$ locus in meningioma tissues.

\section{Methods}

The clinical study protocol was approved by the institutional review board at Dokkyo Medical University with written informed consent obtained from all patients. To assess somatic alterations in the index case, we applied two nodules from adrenal glands, and three regions dissected from a brain tumor. Procedures of gene analysis, as well as immunohistochemical staining, are described in Supplementary Appendix (see section on supplementary material given at the end of this article).

\section{Case description}

A 65-year-old female, who had a 9-year history of hypertension, was referred to our hospital because of hemi-facial spasm and convulsion. A brain MRI indicated a $2.5 \mathrm{~cm}$ diameter dural-based tumor on the right frontal convexity (Fig. 1A). Preoperative laboratory examination indicated that she was diabetic with plasma HbA1c, 8.4\%, and high blood glucose, $218 \mathrm{mg} / \mathrm{dL}$. The brain tumor was completely resected, followed by a pathological diagnosis of angiomatous and microcystic meningioma (Fig. 1B).

Since she was hypertensive and diabetic, abdominal CT was performed, resulting in an unexpected finding of bilateral adrenal hyperplasia (Fig. 1C). Although physical examination showed no typical Cushingoid appearance, results of blood test, as well as challenge tests, were in line with a diagnosis of CS (Supplementary Tables 1 and 2). Adrenal imaging demonstrated noticeable bilateral uptake of ${ }^{131}$ I-iodomethylnorcholesterol (Fig. 1D). Those clinical results were consistent with the diagnosis of PBMAH, validated by pathology after bilateral adrenalectomy (Fig. $1 \mathrm{E}$ and $\mathrm{F})$.

Since both of her children, a 39-year-old male (III-1 in Fig. 1G) and a 36-year-old female (III-2 in Fig. 1G), were hypertensive with bilateral adrenal hyperplasia, genetic testing for ARMC5 was performed in the proband, as well as two of her children, consistently resulting in a germline heterozygous alteration of p.R267* by c.799C $>\mathrm{T}$ among them (Fig. 1G).

Somatic alterations of ARMC5 were then evaluated in brain tumor tissues resected from the proband.
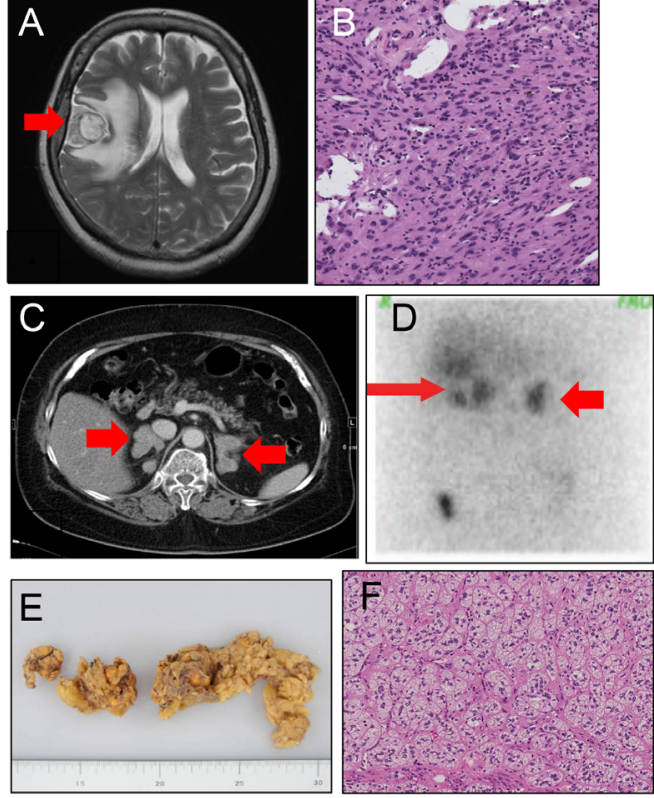

G ।

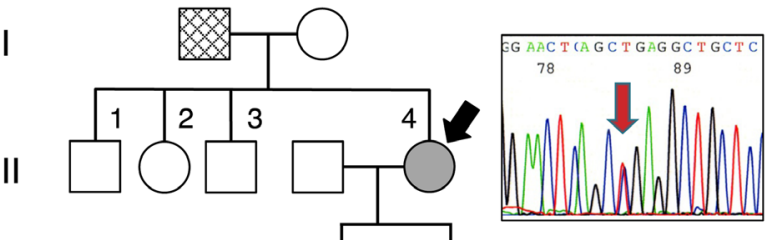

III
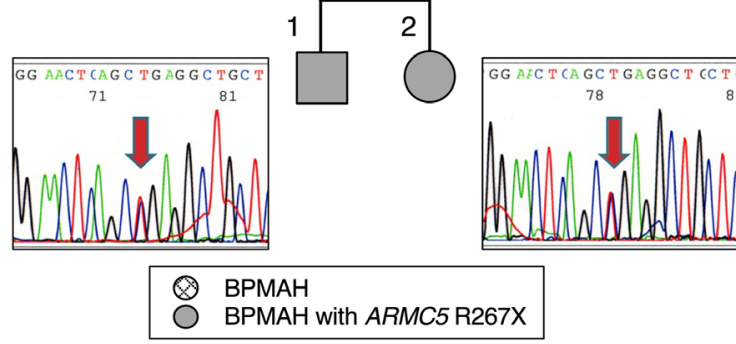

BPMAH with ARMC5 R267X

\section{Figure 1}

Imaging and pathology of the brain tumor. (A) T2-weighted brain MRI scan. The arrow indicates meningioma $(\sim 2.5 \mathrm{~cm}$ diameter) with surrounding edema. (B) Hematoxylin and eosin staining of a dissected tumor section, photographed with a 40-fold objective lens. (C, D, E and F) Imaging and pathology of adrenal glands. (C) Contrast-enhanced CT indicating bilateral macronodular adrenal hyperplasia, as shown by red arrows. (D) Adrenal scintigraphy indicating bilateral uptake of

${ }^{131} 1$-iodomethylnorcholesterol. (E) The left adrenal gland resected with adipose tissues. (F) Hematoxylin and eosin staining of a dissected tumor section, photographed with a 40-fold objective lens. (G) Pedigree of the studied family. Clinical status is indicated by open symbol (unaffected), solid symbol (affected), or oblique symbol (affected but not genotyped). The proband is indicated by a black arrow. Electropherograms indicate the germline heterozygous alteration in exon 3 of ARMC5, c.799C > T, which brings about p.R267*. 
A

\begin{tabular}{|c|c|c|c|}
\hline $16 \mathrm{p}$ & $28 \mathrm{Mbp}$ & 0.2 Mbp & ARMC5 \\
\hline
\end{tabular}

B

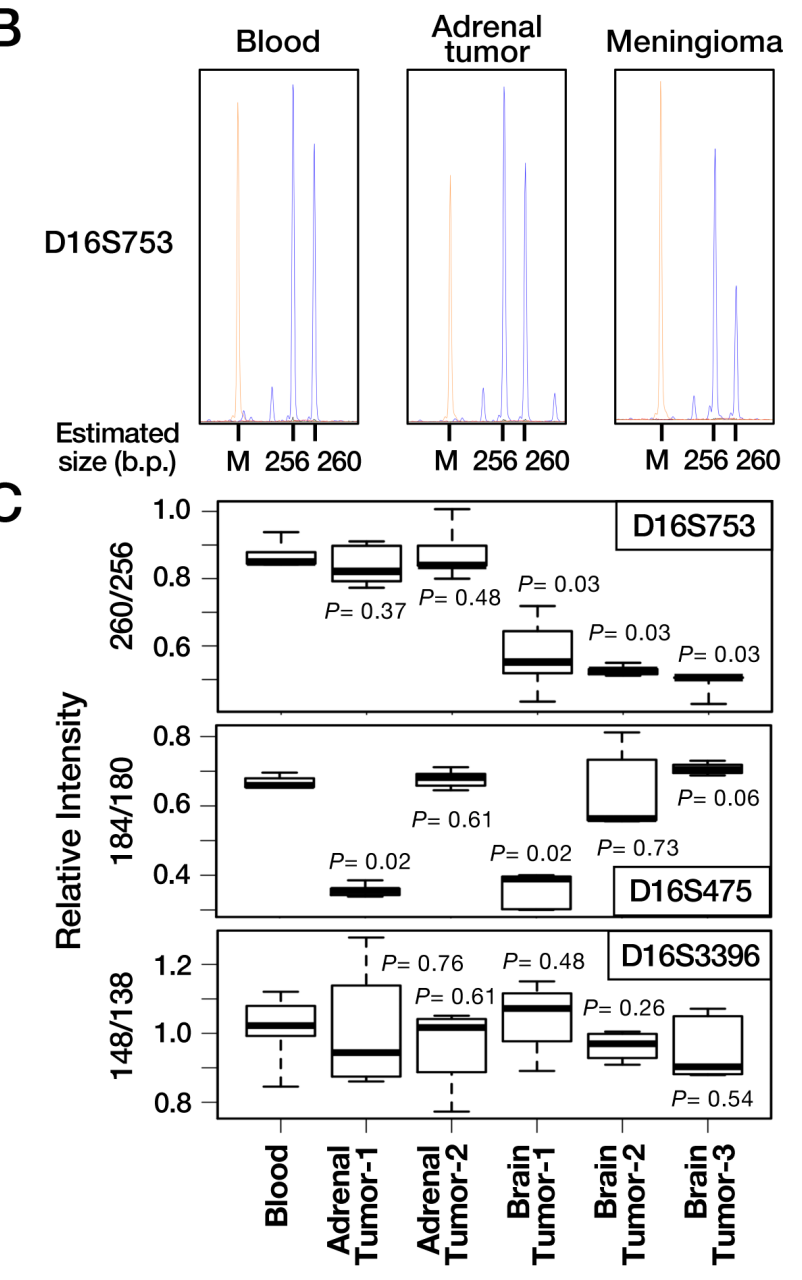

Figure 2

$\mathrm{LOH}$ analysis around the locus of ARMC5. Tissue specimens dissected from three parts of the brain tumor and two respective nodules of adrenal glands, as well as peripheral blood cells, were applied. Fluorescent-labeled PCR products around three microsatellites were separated by capillaryelectrophoresis, and each peak area was quantified by GeneMapper ${ }^{\circledR}$ software. (A) Locations of the tested microsatellites. Note that the schematic drawing may not reproduce the exact scale. Mbp, mega base pairs.
Microsatellite analysis of a short tandem repeat, D16S753, mapped on the locus of ARMC5, 16p11 (Fig. 2A), indicated that allelic ratios of the heterozygous D16S753 in all of the tested brain tumor samples were significantly reduced (from 0.56- to 0.66-fold, $P<0.05$ ), compared to unaffected blood cells (Fig. 2B and C). Genomic sequencing in brain tumors indicated a major peak of thymine overlapped with a minor peak of the unaffected cytosine, likely derived from the significant contamination of monocytes (9) (Supplementary Figure), at nucleotide position 799 in the coding DNA sequence (Fig. 3A). RNA sequencing analysis actually indicated homozygous c.799C $>\mathrm{T}$ in the brain tumor (Fig. 3B). Those results demonstrated that the meningioma harbored LOH at the locus of ARMC5.

In contrast to the brain tumor, the heterozygosities both for the c.799C > T (Fig. 3C and D) and at D16S753 (Fig. 2) were demonstrated in the adrenocortical nodules tested. Furthermore, genomic DNA sequencing in adrenocortical tumors indicated no additional pathogenic variant other than c.799C $>\mathrm{T}$ in the coding sequence of ARMC5 (data not shown). Another somatic alteration, such as genomic variants in UTR or intronic sequence of $A R M C 5$ or alteration of another tumorigenic gene, could be involved in the adrenocortical hyperplasia.

Analysis of another microsatellite marker D16S475 indicated an allelic loss at 16p13.3, 28 mega-bases from $A R M C 5$, in one of three specimens of the brain tumor, as well as one of two adrenal tumors (Fig. 2), while LOH at D16S3396 on 16q was consistently absent in both brain tumor specimens and adrenal tumors (Fig. 2). These results indicated heterogeneity of $\mathrm{LOH}$ pattern even within the single brain tumor: some region likely harbored a focal LOH at 16p11.2, another had a predictively larger one through $16 \mathrm{p} 11$ to $16 \mathrm{p} 13$ (summarized in Fig. 2A).

(B) Representative electropherograms of D16S753. Fragment sizes were estimated by relative positions of two major blue-colored peaks compared to the GeneScan ${ }^{\mathrm{TM}} 500$ LIZ® Size Standard. M, size marker of 250 bases. (C) Allelic ratios determined by relative intensities of allelic peak. Estimated sizes of the fragments are: 256 and 260 in D16S753 (expected range, 252-276, according to the NCBI probe); 180 and 184 in D16S475 (160-189); 138 and 148 in D16S3396 (138-157). $P$ values from Mann-Whitney U-test, when compared to the group of blood, are shown ( $n=4-6$, number of sections analyzed from the tumors, or quadruplicate to sextuplicate measurements with blood sample). 
Genomic Sequence

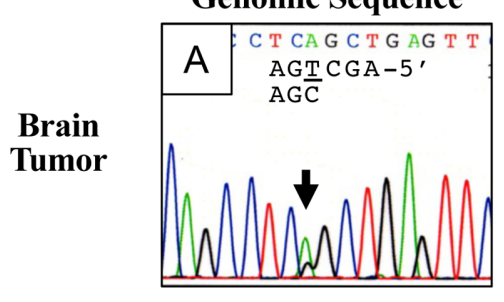

Genomic Sequence

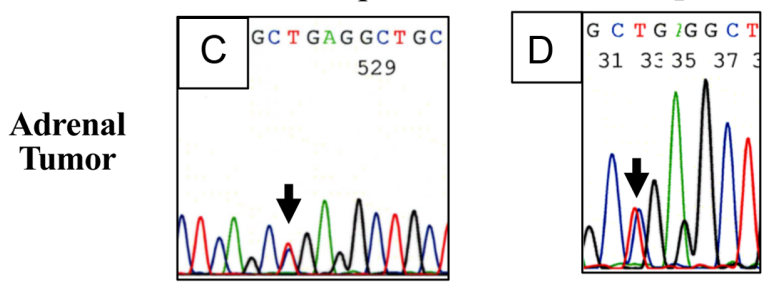

$\mathrm{E}$

\section{Figure 3}

The somatic alteration of $A R M C 5$ in resected tumors.

Electropherograms of the lower strand in meningioma ( $A$ and $B$ ) and those of the upper strand in adrenal tumor ( $C$ and $D)$ are shown. (A) Genomic sequencing of the exon 3 with FFPE specimens. The black arrow indicates a nonsense alteration of c.799C > T as a major peak (green), overlapped by a minor peak (black) of unaffected sequence. (B) mRNA sequencing with FFPE specimens, indicating the homozygous c.799C>T. (C) Genomic sequencing, indicating a heterozygous nonsense alteration of c.799C>T. (D) mRNA sequencing, showing the consistent heterozygous alteration. (E) Distribution of pathogenic variants of $A R M C 5$ reported in patients with both $\mathrm{PBMAH}$ and meningioma $(3,4,5)$. The red arrow corresponds to the pathogenic variant detected in this study. Germline variants are indicated with wrapped text, variants detected in meningioma with red color, and somatic variants in adrenal tumor with black color, respectively (Supplementary Table 3). BTB, Bric-a-Brac, Tramtrack, Broad-complex/Pox virus and Zinc finger (POZ) domain. Note that p.T503fs, derived from c.1506_1507delCA, has been described as p.R502fs in the original reference (5).

\section{Discussion}

We report here a case of PBMAH carrying a heterozygous germline alteration of ARMC5, p.R267* with concomitant meningioma exhibiting LOH for ARMC5. Together with a previous report (5), our case strongly suggested involvement of ARMC5 in the development of meningioma via the two-hit mechanism, as is already well-established in adrenocortical hyperplasia (2).

Several susceptibility genes for familial meningioma have been reported. Of those, pathogenic variants in the NF2 tumor suppressor gene and loss of chromosome 22q, carrying NF2, are the most common genetic alterations associated with the development of meningiomas (10). A study of chromosomal imbalances in 59 cases of sporadic meningioma has indicated high frequency $(\sim 58 \%)$ of loss on 22q, carrying NF2, while loss on $16 \mathrm{p}$, carrying $A R M C 5$, was only $\sim 3 \%$ (11). Interestingly, frequency of gain on $16 \mathrm{p}$ was relatively high $(\sim 20 \%)$, suggesting instability of $16 \mathrm{p}$ in meningioma. Frequency of germline alteration of $A R M C 5$ in familial meningioma is to be elucidated.

A literature search of PubMed (https://www.ncb i.nlm.nih.gov/pubmed/) indicated that three pedigrees carrying pathogenic variants of $A R M C 5$ has been reported to be complicated with meningioma $(3,4,5)$ (summarized in Supplementary Table 3). In four of five previous cases of PBMAH with meningioma $(3,4)$, brain tumor was identified as a secondary incidentaloma by imaging studies for evaluation of the pituitary gland. In contrast, the proband reported in the present study was initially diagnosed as meningioma with neurological symptoms, followed by the diagnosis of PBMAH. In a previous case with abnormal ARMC5, PBMAH was diagnosed 29 years later than that of meningioma (Supplementary Table 3) (4). Those cases have indicated that germline alterations of ARMC 5 can be causative for multiple tumor syndrome, even with meningioma as the primary tumor, and adrenal tumors secondarily developed. Since PBMAH often lacks a typical cushingoid appearance, familial occurrence of meningioma, followed by onset of hypertension and diabetes, may suggest genetic alterations in ARMC5.

ARMC5 contains the Broad-Complex, Tramtrack and Bric a Brac (BTB) domain, predictively important for protein/protein interaction, including dimerization (12). Dysfunction of many BTB-containing proteins are associated with tumorigenesis (13). Three of four pathogenic ARMC5 variants detected in patients with meningioma, including p.R267* in the proband, theoretically eliminate the BTB domain (Fig. 3E and 
Supplementary Table 3). Development of meningioma, therefore, could be dependent on elimination of the BTB domain, consistent with adrenocortical nodules (14). In contrast, all of previous cases with the germline p.R267* have not been complicated with meningioma $(2,7,8)$, inconsistent to our case. Genotyping of ARMC5, therefore, may not be enough to predict the onset of meningioma.

The majority of cases with PBMAH have demonstrated biallelic alterations of ARMC5 in adrenocortical nodules $(2,5,14)$, as a result of the two hits. In contrast, our analysis in adrenocortical nodules indicated only the monoallelic p.R267*, but no somatic alteration detected together. Of note, our Sanger analysis was limited to the coding sequence, and chromosomal microdeletion or gene expression level were not evaluated yet. A post-transcriptional event in the regulation of ARMC5 expression, microdeletion that does not contain D16S753 utilized for our $\mathrm{LOH}$ analysis, and/or alteration of another tumor-related gene(s), therefore, would possibly be associated as the second alteration(s). A dilution by contamination of normal tissues could also explain the discrepancy, although the pathology would not support it (Fig. 1F and Supplementary Fig.). The absence of secondary somatic alteration in adrenal tissues has been reported in some of PBMAH $(14,15)$.

Our case of PBMAH with concomitant meningioma provided findings of the secondary somatic alteration of ARMC5 in meningioma, compatible with the twohit mechanism of tumor formation. To investigate hypertensive patients with a history of meningioma for the presence of CS is highly recommended. In addition, cranial imaging should be offered to patients with PBMAH to exclude a coexisting meningioma. Genetic testing for ARMC5 germline variants might be useful for the identification of affected relatives.

\section{Supplementary materials}

This is linked to the online version of the paper at https://doi.org/10.1530/ EJE-20-0014.

\section{Declaration of interest}

The authors declare that they have no known competing financial interests or personal relationships that could have appeared to influence the work reported in this paper.

\section{Funding}

This research did not receive any specific grant from any funding agency in the public, commercial or not-for-profit sector.

\section{Acknowledgments}

The authors are grateful to their patient and her family members who agreed to participate in this study.

\section{References}

1 Drougat L, Espiard S \& Bertherat J. Genetics of primary bilateral macronodular adrenal hyperplasia: a model for early diagnosis of Cushing's syndrome? European Journal of Endocrinology 2015173 M121-M131. (https://doi.org/10.1530/EJE-15-0532)

2 Assie G, Libe R, Espiard S, Rizk-Rabin M, Guimier A, Luscap W, Barreau O, Lefevre L, Sibony M, Guignat L et al. ARMC5 mutations in macronodular adrenal hyperplasia with Cushing's syndrome. New England Journal of Medicine 2013369 2105-2114. (https://doi. org/10.1056/NEJMoa1304603)

3 Lee S, Hwang R, Lee J, Rhee Y, Kim DJ, Chung UI \& Lim SK. Ectopic expression of vasopressin $\mathrm{V} 1 \mathrm{~b}$ and $\mathrm{V} 2$ receptors in the adrenal glands of familial ACTH-independent macronodular adrenal hyperplasia. Clinical Endocrinology 200563 625-630. (https://doi.org/10.1111/ j.1365-2265.2005.02387.x)

4 Alencar GA, Lerario AM, Nishi MY, Mariani BM, Almeida MQ, Tremblay J, Hamet P, Bourdeau I, Zerbini MC, Pereira MA et al. ARMC5 mutations are a frequent cause of primary macronodular adrenal hyperplasia. Journal of Clinical Endocrinology and Metabolism 201499 E1501-E1509. (https://doi.org/10.1210/jc.2013-4237)

5 Elbelt U, Trovato A, Kloth M, Gentz E, Finke R, Spranger J, Galas D, Weber S, Wolf C, Konig K et al. Molecular and clinical evidence for an ARMC5 tumor syndrome: concurrent inactivating germline and somatic mutations are associated with both primary macronodular adrenal hyperplasia and meningioma. Journal of Clinical Endocrinology and Metabolism 2015100 E119-E128. (https://doi.org/10.1210/ jc.2014-2648)

6 Berthon A, Faucz F, Bertherat J \& Stratakis CA. Analysis of ARMC5 expression in human tissues. Molecular and Cellular Endocrinology 2017441 140-145. (https://doi.org/10.1016/j.mce.2016.08.018)

7 Gagliardi L, Schreiber AW, Hahn CN, Feng J, Cranston T, Boon H, Hotu C, Oftedal BE, Cutfield R, Adelson DL et al. ARMC5 mutations are common in familial bilateral macronodular adrenal hyperplasia. Journal of Clinical Endocrinology and Metabolism 201499 E1784-E1792. (https://doi.org/10.1210/jc.2014-1265)

8 Espiard S, Drougat L, Libe R, Assie G, Perlemoine K, Guignat L, Barrande G, Brucker-Davis F, Doullay F, Lopez S et al. ARMC5 mutations in a large cohort of primary macronodular adrenal hyperplasia: clinical and functional consequences. Journal of Clinical Endocrinology and Metabolism 2015100 E926-E935. (https://doi. org/10.1210/jc.2014-4204)

9 Domingues PH, Teodosio C, Ortiz J, Sousa P, Otero A, Maillo A, Barcena P, Garcia-Macias MC, Lopes MC, de Oliveira C et al. Immunophenotypic identification and characterization of tumor cells and infiltrating cell populations in meningiomas. American Journal of Pathology 2012181 1749-1761. (https://doi.org/10.1016/j. ajpath.2012.07.033)

10 Riemenschneider MJ, Perry A \& Reifenberger G. Histological classification and molecular genetics of meningiomas. Lancet: Neurology 20065 1045-1054. (https://doi.org/10.1016/S14744422(06)70625-1)

11 Weber RG, Bostrom J, Wolter M, Baudis M, Collins VP, Reifenberger G \& Lichter P. Analysis of genomic alterations in benign, atypical, and anaplastic meningiomas: toward a genetic model of meningioma progression. PNAS 199794 14719-14724. (https://doi.org/10.1073/pnas.94.26.14719)

12 Bardwell VJ \& Treisman R. The POZ domain: a conserved proteinprotein interaction motif. Genes and Development 19948 1664-1677. (https://doi.org/10.1101/gad.8.14.1664) 
13 Kelly KF \& Daniel JM. POZ for effect - POZ-ZF transcription factors in cancer and development. Trends in Cell Biology 200616 578-587. (https://doi.org/10.1016/j.tcb.2006.09.003)

14 Correa R, Zilbermint M, Berthon A, Espiard S, Batsis M, Papadakis GZ, Xekouki P, Lodish MB, Bertherat J, Faucz FR et al. The ARMC5 gene shows extensive genetic variance in primary macronodular adrenocortical hyperplasia. European Journal of Endocrinology 2015173 435-440. (https://doi.org/10.1530/EJE-15-0205)

15 Liu Q, Tong D, Xu J, Yang X, Yi Y, Zhang D, Wang L, Zhang J, Zhang Y, Li Y et al. A novel germline ARMC5 mutation in a patient with bilateral macronodular adrenal hyperplasia: a case report. BMC Medical Genetics 201819 49. (https://doi.org/10.1186/s12881-018-0564-2)

Received 9 January 2020

Revised version received 12 August 2020

Accepted 29 September 2020 